\title{
Two new species of the genus Teuchophorus (Diptera: Dolichopodidae) from Taiwan
}

\author{
Mengqing Wang, Ding Yang*, \& Patrick Grootaert
}

Wang, M., Ding, Y. \& Grootaert, P. 2006: Two new species of the genus Teuchophorus (Diptera: Dolichopodidae) from Taiwan. - Entomol. Fennica 17: 105-109.

Two species are described as new to science from Taiwan: Teuchophorus elongatus $\mathrm{sp}$. $\mathrm{n}$. and T. taiwanensis $\mathrm{sp}$. n. Currently three Teuchophorus species are known from Taiwan. A key is given to help identify the Chinese species.

M. Wang \& D. Yang (author for correspondence), Department of Entomology, China Agricultural University, Beijing 100094, China

P. Grootaert, Department of Entomology, Royal Belgian Institute of Natural Sciences, Vautierstraat 29, B-1000 Brussels, Belgium

Received 2 April 2004, accepted 22 July 2005

\section{Introduction}

The genus Teuchophorus Loew belongs to the subfamily Sympycninae. It is characterized by the following features: crossvein m-cu joining $\mathrm{Cu}$ at a distinctly oblique angle, apical section of $\mathrm{M}_{1+2}$ turned up immediately after m-cu, acr uniseriate or absent (exceptionally biseriate), and male wing usually with costal callus between tips of $\mathrm{R}_{1}$ and $\mathrm{R}_{2+3}$.

Ninety-eight species of the genus Teuchophorus have been recorded in the world so far. Four species are known from the Nearctic Region, 15 from the Palaearctic and eight from the Afrotropical Region, 36 from the Australasian Region, and 35 from the Oriental Region (Dyte 1975, Meuffels \& Grootaert 2004, Negrobov 1991, Olejniček 2003, Yang 2001, Yang \& Saigusa 1999, 2000, 2001), but no species have been reported from the Neotropics. Moreover, only one fossil species is known, viz. T. sobrius from Tanzania (Meuffels \& Grootaert 2004).

A Teuchophorus species was recorded from Taiwan in 1924 as Mastigomyia gratiosa. The male of this species has a very long first flagellomere with a very short thorn-like arista. Meuffels \& Grootaert (2004) regarded Mastigomyia Becker as a junior synonym of Teuchophorus Loew, 1857, but still considered the gratiosus group as a distinct species group within Teuchophorus.

In this paper, two additional species of Teuchophorus Loew from Taiwan are described as new to science. The material was collected by Dr. Ignac Sivec and Dr. Bogdan Horvat with sweep nets in 1996. The type specimens are deposited in the Slovenian Museum of Natural History, Ljubljana, Slovenia. All material is preserved in $75 \%$ alcohol.

The following abbreviations are used: acr $=$ acrostichal, ad $=$ anterodorsal, apv $=$ apicoventral, $\mathrm{av}=$ anteroventral, $\mathrm{dc}=$ dorsocentral, $\mathrm{h}=$ humeral, ih = inner humeral, LI = fore leg, LII = mid leg, LIII = hind leg, $\mathrm{npl}=$ notopleural, oc = ocellar, $\mathrm{pd}=$ posterodorsal, $\mathrm{ph}=$ posthumeral, $\mathrm{psa}$ $=$ postalar, $\mathrm{pv}=$ posteroventral, $\mathrm{sa}=$ supraalar, $\mathrm{su}$ $=$ sutural. 


\section{Taxonomy}

\subsection{Teuchophorus Loew, 1857}

Teuchophorus Loew, 1857: 44. Type species: Dolichopus spininerellus Zetterstedt, 1843 (des. Coquillett, 1910: 613).

Mastigomyia Becker, 1924: 121. Type species: Mastigomyia gratiosa Becker, 1924 (monotypy).

Olegonegrobovia Grichanov, 1995: 125. Type species: Olegonegrobovia zlobini Grichanov, 1995 (original designation).

Generic characters. Body small; thoracic pleura yellowish brown to dark brown; abdominal terga sometimes with green gloss; frons broad, narrowing toward antennae. In male, eyes often contiguous for a short distance on face; first flagellomere more or less conical, sometimes up to five times as long as wide, arista dorsal. 5-6 dc; acr uniseriate or absent (exceptionally irregularly biseriate); 1 h, 1 ph, 1 ih, 1 su, 2 npl, 2 sa, 1 psa; scutellum with 2 pairs of bristles and several marginal hairs. Legs in male could be somewhat modified and (or) adorned, not in female, and hind tarsomere 1 always shorter than tarsomere 2 . Male wing usually with costal callus between tips of $\mathrm{R}_{1}$ and $\mathrm{R}_{2+3}$; crossvein $\mathrm{m}$-cu, joining $\mathrm{Cu}$ at a distinctly oblique angle, apical section of $\mathrm{M}_{1+2}$ turned up immediately after $\mathrm{m}$-cu; anal vein represented by a fold.

\subsection{Key to Chinese Teuchophorus (males)}

1. Costal callus absent

- Costal callus present

2. First flagellomere much longer than wide 3

- First flagellomere as long as wide 5

3. Arista reduced to a stump. Taiwan, Laos, Ryukyu Islands

gratiosus (Becker, 1924)

- Arista developed as usual

4. Arista slightly longer than first flagellomere, and hairless except apex. Sichuan (Emei Mts) ventralis Yang \& Saigusa, 2000

- Arista about 2 times as long as first flagellomere, wholly haired. Taiwan

elongatus sp. n.
5. Six dc present

- Five dc present. Sichuan (Emei Mts) emeiensis Yang \& Saigusa, 2000

6. Eyes distinctly separated on face; body length shorter than $1.5 \mathrm{~mm}$

- Eyes nearly contiguous on face; body length $2.1 \mathrm{~mm}$. Taiwan taiwanensis sp. $\mathrm{n}$.

7. Hind tibia with $2 \mathrm{pd}$, and row of 9-10 av. Yunnan yunnanensis Yang \& Saigusa, 2001

- Hind tibia with 3-4 pd, and 5-7 av. Henan, Sichuan, Korea

sinensis Yang \& Saigusa, 2000

8. First flagellomere longer than wide (1.7 times as long as wide). Zhejiang

tianmushanus Yang, 2001

- First flagellomere wider than long (about 0.7 times as long as wide). Henan

nigrescus Yang \& Saigusa, 1999

\subsection{Teuchophorus elongatus sp. n. (Fig. 1)}

Material examined. Holotype $\widehat{\nearrow}$ : Taiwan: Taipei county, N. Shihpei (435 m a.s.1.), 7.IV.1996, leg. I. Sivec \& B. Horvat (Ljubljana). Paratypes $1 \stackrel{\lambda}{\lambda}, 1$ q: Taiwan: Hualien county, Nanan $(200 \mathrm{~m})$, 5.IV.1996, leg. I. Sivec \& B. Horvat (Ljubljana); 1 + : same data as holotype.

Diagnosis. First flagellomere much elongated and subtriangular, about 3.2 times as long as wide; arista dorsal, with basal segment rather short. Fore and hind tibiae slightly curved.

Description. Male. Wing (Fig. 1a) hyaline, slightly tinged with grey; veins brown, $\mathrm{R}_{4+5}$ and $\mathrm{M}$ slightly curved, weakly convergent apically; $\mathrm{CuAx}$ ratio 0.5. Squama brown with dark margin, and with long black hairs. Halter dark brown with yellow base. Head metallic green with pale gray pollen; eyes contiguous on face. Hairs and bristles on head black; postocular bristles (including posterior ventral hairs) black. Ocellar tubercle weak, with one pair of long oc and 2 very short posterior hairs. Antenna (Fig. 1b) blackish; first flagellomere much elongated and subtriangular, about 3.2 times as long as wide; arista dorsal, brown, with basal segment rather short. Proboscis dark brown with black hairs; palpus blackish with black hairs and 1 black apical spine-like bristle.

Thorax dark metallic green with pale gray 
Fig. 1. Teuchophorus elongatus sp. n., male. a. Wing, dorsal view. $b$. Antenna, lateral view. c. Hind femur and tibia, lateral view. d. Fore tarsus, lateral view. e. Genitalia, lateral view. Scale bar $0.1 \mathrm{~mm}$.

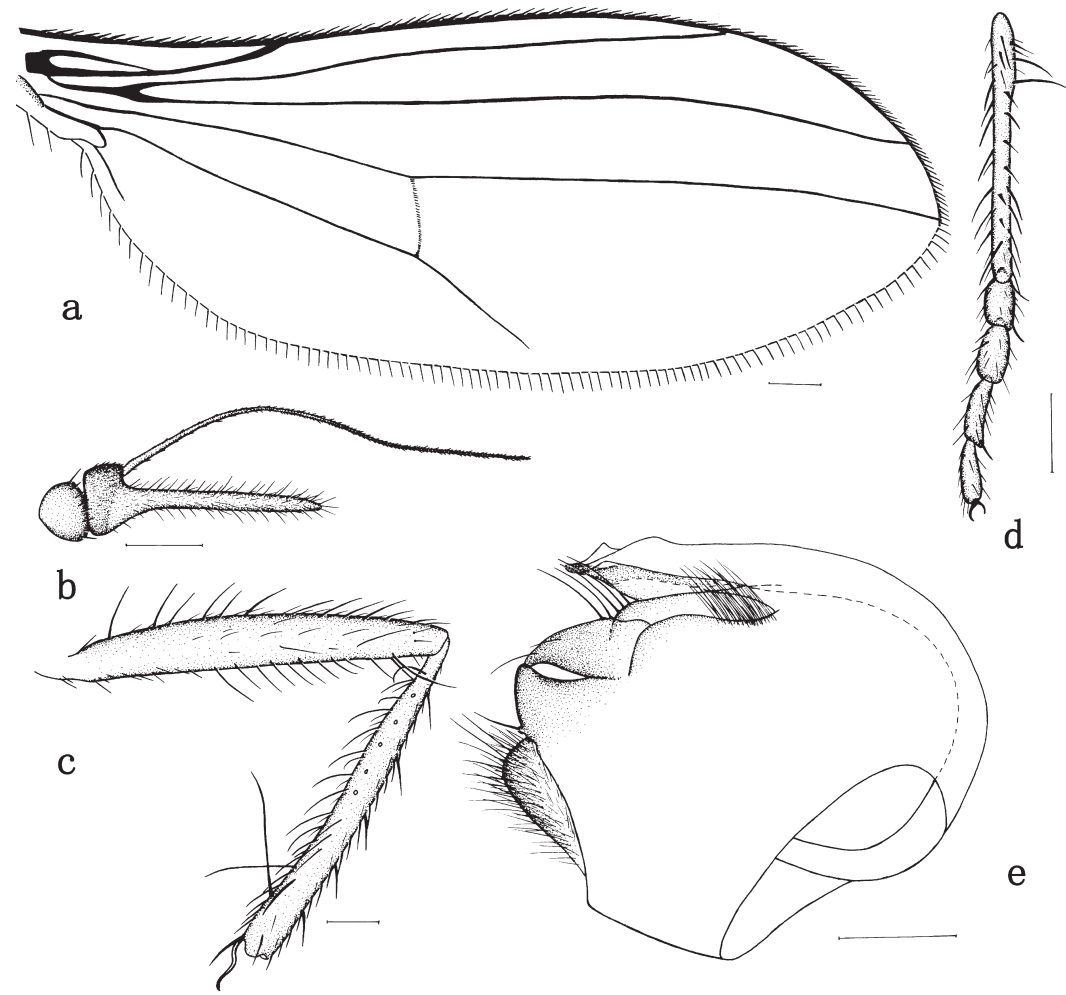

pollen. Hairs and bristles on thorax black; $6 \mathrm{dc}, 6-$ 7 uniseriate acr; 1 h, 1 ph, 1 ih, 1 su, 2 npl, 2 sa, 1 psa; scutellum with 1 pair of bristles and several very short marginal hairs.

Legs dark brown. Hairs and bristles on legs black; fore coxa with 8 anterior bristles, mid coxa with dense anterior hairs, hind coxa with 1 outer bristle at basal $1 / 3$. Fore femur with 3-4 $\mathrm{v}$ at tip; mid femur with 1 rather strong spine-like $v$ at middle and 2 rows of $\mathrm{v}$; hind femur (Fig. 1c) with one irregular row of ad and one row of $\mathrm{v}$ on apical half, apically with 1 av and 1 pv. Fore tibia slightly curved, apically with 1 spine-like bristle; mid tibia with 3 ad near base (of which 1 stronger), 1 row of short v, and 2-3 apical bristles; hind tibia (Fig. $1 \mathrm{c}$ ) slightly curved, with 1 curved $\mathrm{v}$ at base, $5 \mathrm{pd}, 2$ rows of thin v, 2 long bristles on apical $1 / 4$, apically with 1 av and 1 bent spine-like pv. Fore tarsomere 1 (Fig. 1d) much elongated (nearly as long as tarsomeres 2-5), with 1 row of strong ad and 1 row of $\mathrm{v}$ (of which 2 basal $\mathrm{v}$ distinctly longer); tarsomeres 2-3 slightly swollen and subequal in length. Relative lengths of tibia and 5 tarsomeres: LI $2.1: 1.8: 0.35: 0.4: 0.4: 0.4$;
LII $3.5: 1.7: 0.7: 0.6: 0.5: 0.4 ;$ LIII $3.8: 1.2: 1.0$ $: 0.7: 0.5: 0.4$.

Abdomen chiefly metallic green with gray pollen. Hairs and bristles on abdomen black; tergum 1 brown, with one row of long bristles on posterior margin.

Male genitalia (Fig. 1e). Epandrium slightly longer than wide, with one group of (about 20) long soft ventral hairs near base of hypandrium; surstylus with wide dorsal lobe bearing 1 bristle, nearly acute apically, and with lightly narrow ventral lobe acute apically; hypandrium rather short and thick with middle ridge.

Length. Body length 1.9-2.0 mm, wing length 1.9-1.9 mm.

Female. Body length $2.0 \mathrm{~mm}$, wing length 2.1 $\mathrm{mm}$. Similar to male, but legs paler, first flagellomere about 2 times as long as wide.

Remarks. The new species is similar to Teuchophorus ventralis Yang \& Saigusa in having the first flagellomere much longer than wide, but may be separated from the latter by having the rather long arista about 2 times as long as the first flagellomere and haired along its length. In 
ventralis, the arista is just a little longer than the first flagellomere and hairless except the apex (Yang \& Saigusa 2001).

Distribution. Taiwan (Oriental Region).

\subsection{Teuchophorus taiwanensis sp. n. (Fig. 2)}

Material examined. Holotype ${ }^{1}$ : Taiwan: Taipei county, N Shihpei, 435m a.s.1., 7.IV.1996, leg. I. Sivec \& B. Horvat (Ljubljana). Paratype $q$ : same data as holotype.

Diagnosis. First flagellomere nearly triangular, as long as wide; arista dorsal. 6 dc. Surstylus with acute finger-like dorsal lobe, bearing 1 apical bristle, and wide ventral lobe, rather wide with bristles apically, bearing 2 very long ventral bristles near middle; hypandrium rather thick.

Description. Male. Wing (Fig. 2a) hyaline, slightly tinged with greyish; veins brown, $\mathrm{R}_{4+5}$ and $\mathrm{M}$ straight and nearly parallel apically; $\mathrm{CuAx}$ ratio 0.4. Squama brown with dark margin, and with long black hairs. Halter yellow with brown base. Head metallic green, with pale gray pollen; eyes nearly contiguous on face. Hairs and bristles on head black; postocular bristles (including posterior ventral hairs) black. Ocellar tubercle weak, with one pair of long oc and 2 very short posterior hairs. Antenna (Fig. 2b) blackish, first flagellomere nearly triangular, as long as wide; arista [apical segment broken] dorsal, blackish, with basal segment rather short. Proboscis dark brown with black hairs; palpus blackish with black hairs and 1 black spine-like bristle.

Thorax dark metallic green with pale gray pollen. Hairs and bristles on thorax black; $6 \mathrm{dc}, 4$ 5 uniseriate short and hair-like acr; $1 \mathrm{~h}, 1 \mathrm{ph}, 1 \mathrm{ih}$, $1 \mathrm{su}, 2 \mathrm{npl}, 2 \mathrm{sa}, 1 \mathrm{psa}$; scutellum with 2 pairs of bristles (outer pair very short, hair-like) and several very short marginal hairs.

Legs brownish yellow. Hairs and bristles on legs black; fore coxa with 6 apical bristles, hind coxa with 1 outer bristle at basal 1/3. Hind femur

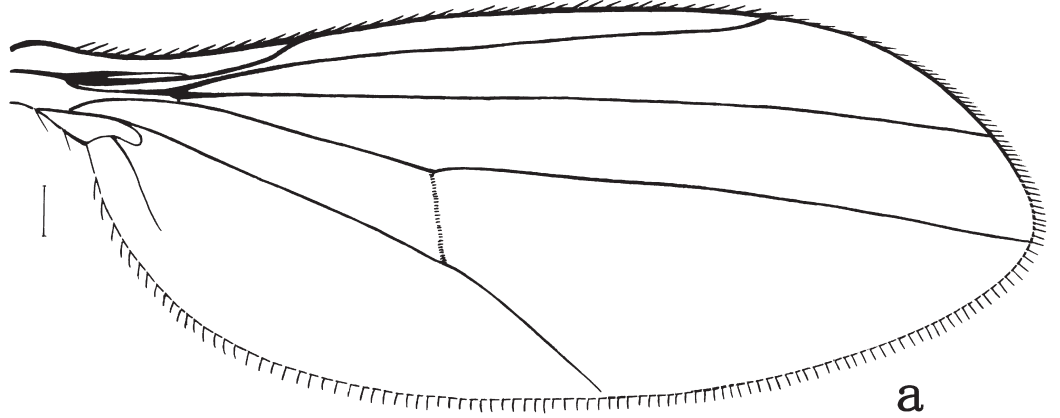

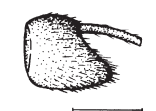

b

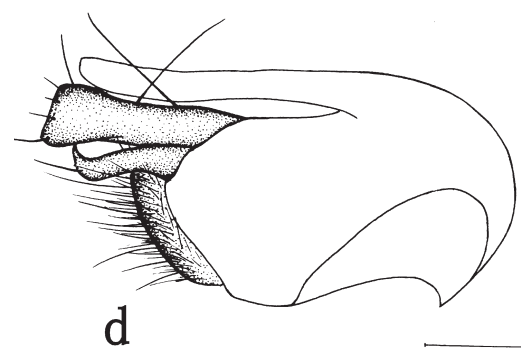

e

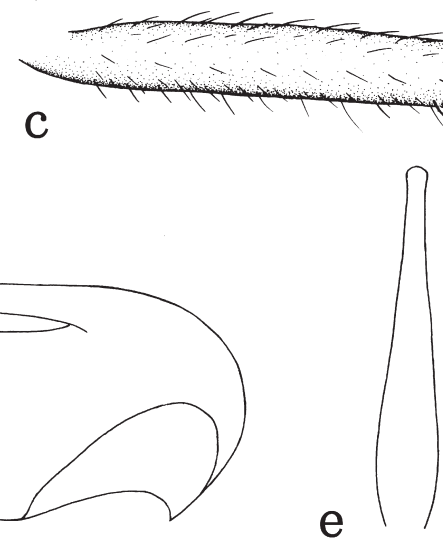

Fig. 2. Teuchophorus taiwanensis sp. n., male. a. Wing. b. Antenna, lateral view. c. Hind femur and tibia, lateral view. d. Genitalia, lateral view. e. Hypandrium, ventral view. Scale bar $0.1 \mathrm{~mm}$. 
(Fig. 2c) with 2 rows of short erected v, and 2 long apical av. Fore tibia apically with 1 short pv; mid tibia with 2 ad, 1 pd, and 2 apical bristles; hind tibia with $5 \mathrm{pd}, 1$ row of 10 long $\mathrm{v}$, and 2 apical bristles. Hind tarsomere 1 shorter than tarsomere 2. Relative lengths of tibia and 5 tarsomeres: LI $2.0: 1.2: 0.5: 0.4: 0.3: 0.3 ;$ LII $3.1: 1.3: 0.7: 0.6$ $: 0.4: 0.3 ;$ LIII $3.2: 0.7: 1.0: 0.6: 0.5: 0.3$.

Abdomen chiefly metallic green with gray pollen. Hairs and bristles on abdomen black; tergum 1 brown with one row of long bristles on posterior margin, sterna $2-5$ with long soft ventral hairs.

Male genitalia (Figs. 2 d-e). Epandrium distinctly longer than wide, its surstylus with acute finger-like dorsal lobe, bearing 1 apical bristle, and wide ventral lobe, rather wide apically with bristles, bearing 2 very long ventral bristles near middle; hypandrium rather thick.

Length. Body length $2.1 \mathrm{~mm}$, wing length 2.0 $\mathrm{mm}$.

Female. Body length $2.0 \mathrm{~mm}$, wing length 2.1 mm. Similar to male.

Remarks. The new species is much similar to Teuchophorus sinensis Yang \& Saigusa in having the first flagellomere as long as wide, but may be separated from the latter by having the nearly contiguous eyes and hind tibia with 5 pd and 1 row of $10 \mathrm{v}$. In sinensis, the eyes are distinctly separated, the hind tibia has 3-4 pd and 1 row of 5-7 v (Yang \& Saigusa 1999).

Distribution. Taiwan (Oriental Region).

Acknowledgements. We are grateful to Dr. I. Sivec and B. Horvat (Ljubljana) for providing the specimens. This research was supported by the National Natural Science Foundation of China (No. 30225009), and 2004-2005 Sino-Slovenian Scientific and Technological Cooperative Program.

\section{References}

Becker, T. 1924: Dolichopodidae from Formosa. Nachtrag zu den dipterologischen Studien 'Dolichopodidae der Indo-Australischen Region'. — Zool. Meded. (Leiden) $8: 120-131$.

Dyte, C. E. 1975: Family Dolichopodidae. - In: Delfinado, M. D. \& Hardy, D. E. (eds.), A Catalog of the Diptera of the Oriental Region 2: 212-258. The University Press of Hawaii, Honolulu. 459pp.

Grichanov, I. Ya. 1995: Olegonegrobovia (Diptera: Dolichopodidae), new genus from Uganda. - An International Journal of Dipterological Research. 6: 125-128.

Loew, H. 1857: Die Familie der Dolichopoden. Neue Beiträge zur Kenntniss der Dipteren. Fünfter Beitrag. - Programm K. Realschule Meseritz 1857: 1-56.

Meuffels, H. \& Grootaert, P. 2003 (2004): The genus Teuchophorus in South-East Asia and New Guinea, description of new species, species-groups and their phylogeny (Insect, Diptera, Dolichopodidae). - J. Nat. Hist. 38: 143-258.

Negrobov, O. P. 1991: Family Dolichopodidae. — In: Soós Á. \& Papp, L. (eds.), Catalogue of Palaearctic Diptera 7: 51-64. Akademiai Kiado, Budapest. 291 pp.

Olejniček, J. 2003: Some Dolichopodidae (Diptera) from Laos. - Biologia 58 (2): 191-200.

Yang, D. 2001: Diptera: Dolichopodidae. - In: Wu, H. \& Pan, C. (eds.), Insects of the Tianmushan Mountain: 428-441. Science Press, Beijing. 764 pp. [In Chinese.]

Yang, D. \& Saigusa, T. 1999: New species of Dolichopodidae from Henan (Diptera: Empidoidea). - In: Shen, X. \& Shi, Z. (eds.), The Fauna and Taxonomy of Insects in Henan 4: 189-209. China Agricultural Scientech Press, Beijing. 415 pp. [In Chinese.]

Yang, D. \& Saigusa, T. 2000: New and little known species of Dolichopodidae from China (VII): Diptera from Emei Mountain (2). - Bull. Inst. Royal Sci. Nat. Belg. Ent. 70: 219-242.

Yang, D. \& Saigusa, T. 2001: New and little known species of Dolichopodidae (Diptera) from China (IX). - Bull. Inst. Royal Sci. Nat. Belg. Ent. 71: 165-188. 